This item was submitted to Loughborough's Research Repository by the author.

Items in Figshare are protected by copyright, with all rights reserved, unless otherwise indicated.

\title{
Determination of overlap in lidar systems
}

PLEASE CITE THE PUBLISHED VERSION

http://dx.doi.org/10.1364/AO.50.005791

\section{PUBLISHER}

(C) Optical Society of America

\section{VERSION}

VoR (Version of Record)

\section{LICENCE}

CC BY-NC-ND 4.0

\section{REPOSITORY RECORD}

Hey, Joshua D. Vande, Jeremy M. Coupland, Ming Hui Foo, James Richards, and Andrew P. Sandford. 2019. "Determination of Overlap in Lidar Systems". figshare. https://hdl.handle.net/2134/13308. 


\title{
Determination of overlap in lidar systems
}

\author{
Joshua Vande Hey, ${ }^{1, *}$ Jeremy Coupland, ${ }^{1}$ Ming Hui Foo, ${ }^{2}$ \\ James Richards, ${ }^{2}$ and Andrew Sandford ${ }^{2}$ \\ ${ }^{1}$ Wolfson School of Mechanical and Manufacturing Engineering, Loughborough University, \\ Ashby Road, Loughborough, Leicestershire, LE11 3TU, UK \\ ${ }^{2}$ Campbell Scientific, Ltd., 80 Hathern Road, Shepshed, Loughborough, Leicestershire, LE12 9GX, UK \\ ${ }^{\star}$ Corresponding author: mmjdv@Iboro.ac.uk
}

Received 18 May 2011; accepted 17 July 2011; posted 22 July 2011 (Doc. ID 147746); published 12 October 2011

\begin{abstract}
The overlap profile, also known as crossover function or geometric form factor, is often a source of uncertainty for lidar measurements. This paper describes a method for measuring the overlap by presenting the lidar with a virtual cloud through the use of an imaging system. Results show good agreement with horizontal hard target lidar measurements and with geometric overlap calculated for the ideal aberration-free case. (C) 2011 Optical Society of America

OCIS codes: $\quad 010.3640,120.4570,280.3640$.
\end{abstract}

\section{Introduction}

Lidar measurements of atmospheric constituents were first carried out by Ligda [1] and Fiocco and Grams [2] who made time-resolved intensity measurements of light elastically backscattered from the atmosphere as laser pulses propagated through it. For the case of weak, elastic scattering from atmospheric aerosols, the backscattered intensity is related to the transmission and backscatter coefficients by the lidar equation. Under the assumption that the effects of multiple scattering are negligible, the lidar equation in its simplest form [3] expresses the backscattered signal power, $P$, as a function of target range, $R$, such that

$$
P(R)=K G(R) \beta(R) T(R),
$$

where $K$ is the system efficiency factor, $\beta(R)$ is the backscatter coefficient at distance $R$, and $T(R)$ is the transmission term that describes round-trip losses as the laser pulse travels through the atmosphere to and from range $R$. The remaining factor, $G(R)$, the geometric factor, is the concern of this paper and is generally stated as

0003-6935/11/305791-07\$15.00/0

(C) 2011 Optical Society of America

$$
G(R)=\frac{O(R)}{R^{2}},
$$

where $O(R)$ is the overlap function describing the fraction of the laser beam cross section contained by the receiver field of view as a function of range. In this expression, the quadratic factor in the denominator is due to the reduction in the solid angle subtended by the lidar receiver and is consequently inversely proportional to the square of the distance from the target to the entrance pupil [3]. When a lidar is in normal use it is noted that this distance is simply equal to the range; however, if additional optics are included between the lidar and the target, the position of the entrance pupil changes, and for this reason in this paper it is more useful to write the geometrical factor in the form

$$
G(R)=\frac{O(R)}{R_{p}^{2}},
$$

where $R_{p}$ is the distance from the target to the entrance pupil.

In order to calculate $\beta(R)$ and $T(R)$ from lidar data, inversion of the lidar equation is necessary. This is commonly implemented using the process originally proposed by Klett [4] , and in order for this to be done 
correctly it is clearly important to know the overlap profile $O(R)$ of the transmitted laser beam with the receiver field of view [5]. It is possible to make the transmitter beam and receiver field of view nearly coincident and therefore attempt to make the overlap function identically 1 by using a coaxial arrangement with common optics such that the laser and detector use exactly the same aperture. One problem with this approach is that it is difficult to ensure that the overlap function is identically 1 because of nonuniform angular and spatial distributions of laser power and/or detector sensitivity [6]. In addition, such a lidar system is difficult to build in practice while avoiding detector saturation due to laser light reflected directly back from the optics. For this reason most lidar systems use biaxial optics [7], expand the laser beam outside of the receiver optics [8], or utilize separate regions of the aperture for the laser and receiver [9]. Campbell et al. [10] used a coaxial common optics approach where in order to limit optical feedback, the receiver field-stop is placed on the detector side of the beam splitter that directs the laser beam out of the telescope. All three of these configurations require overlap correction to some extent, so knowledge of the overlap function is necessary for calibration of their measurements at near ranges.

If the overlap function is not accurately known, then estimates of the aerosol backscatter coefficient at low altitudes will be poor [11]. For this reason, lidar systems frequently invert the lidar equation using only data collected beyond the point where there is high confidence that full overlap has been reached. If overlap can be predicted, however, more accurate estimates of aerosol distributions and derived parameters such as vertical visibility in the lower atmosphere can be made.

Theoretical determination of the overlap function has been discussed by several authors. Geometrical optics has been employed analytically [12-16], through ray tracing $[17,18]$, and in a hybrid approach along with diffraction theory [19]. These theoretical techniques are extremely useful for lidar system design; however, the success of these approaches for overlap calibration of a specific instrument in the field relies on reliable knowledge of system parameters. Such parameters include the laser source power distribution and propagation, the angular and positional response of the detector, and the performance of the optics, which are often difficult to obtain in practice [20].

A number of experimental techniques applicable to elastic backscatter lidar have utilized atmospheric measurements to determine overlap. Sasano et al. [11] used measurements of a well-mixed boundary layer, and Tomine et al. [21] used measurements of a light mist to determine the overlap. Dho et al. [22] considered data obtained beyond the point of full overlap and used a polynomial fit to extrapolate the atmospheric conditions below this point, which were then used to determine the overlap function. All of these measurements rely on accurate estimates of atmospheric conditions that are difficult to verify in practice. Related techniques for Raman lidar were developed by Wandinger and Ansmann [20], who presented an experimental method that uses a Raman molecular channel and an elastic channel during lidar measurements and determines the overlap function from the two channels. Hu et al. [23] also presented a Raman lidar method, based on comparison of Raman returns with radiosonde or atmosphere model data. Neither of these techniques, however, are applicable to lidar systems that measure only elastic returns. Recently, a modification of the method proposed by Wandinger and Ansmann [20] applicable to elastic lidar was presented by Guerrero-Rascado et al. [24]. This approach determined the overlap of $106 \overline{4 \mathrm{~nm}}$ elastic lidar systems by comparing returns from these systems with returns from colocated ceilometer systems. This method, however, relies on the assumption that the comparison ceilometers have full and stable overlap starting from a range of $30 \mathrm{~m}$ and requires measurement or estimation of the extinction-to-backscatter ratio of the atmosphere used for comparison.

In this paper an optical test system capable of measuring the overlap of a lidar system is proposed. The optical test system can be considered as a means of presenting a virtual cloud that appears at a prescribed distance from the lidar instrument. The overlap region is characterized by positioning the virtual cloud at various effective ranges and measuring the lidar returns from each range. The results of the measurement are compared with horizontal lidar returns from a hard target translated along the overlap region and with the overlap function calculated using geometrical optics.

\section{Theory of Overlap Measurement}

The method we propose to measure lidar overlap is similar in concept to placing a planar target with isotropic scattering properties at various distances from the instrument and measuring the lidar return from each distance. This method is impractical; however, since a long (up to several kilometers) unobstructed path with homogeneous atmospheric properties is required, and since, depending on the laser beam divergence angle, a large target may be required at the far end of the measurement range. To overcome these practical difficulties, an optical system is used to present at various ranges a virtual image of the target to the instrument. The simplest configuration that is required to achieve this is presented in Fig. 1.

In this configuration, an imaging lens outside the lidar system is used to present a virtual image of a scattering target to the instrument. Using the Gaussian lens law [25] it is straightforward to show that this image appears at a distance, $R$, from the instrument given by

$$
R\left(a_{o}\right)=\omega+\left|\frac{f_{a} a_{o}}{a_{o}-f_{a}}\right|,
$$




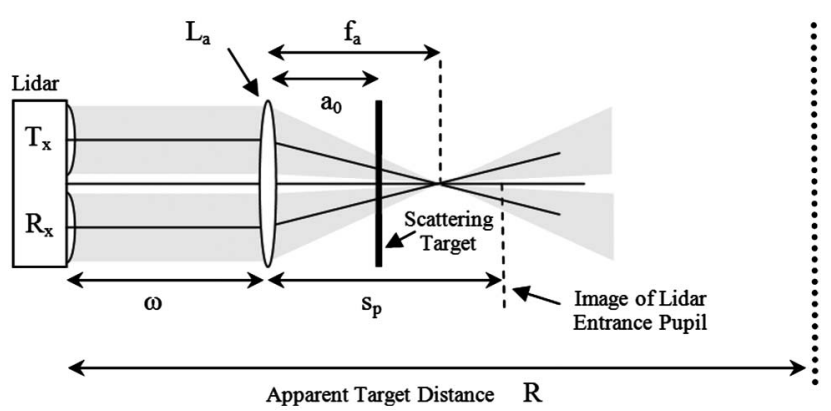

Fig. 1. Imaging system.

where the constant $\omega$ is the distance from the lidar to the imaging lens, $f_{a}$ is the focal length of the imaging lens, and $a_{o}$ is the distance from the imaging lens to the target. It is clear that when the target is in the focal plane its image is presented at infinity. It is important to realize that although $s$ is the distance at which the image of the target is presented to the instrument, it cannot be simply substituted as a range into the geometric factor $G(R)$ in Eq. (2). This is because, as noted previously, the light collected by the lidar is proportional to the inverse square of the distance from the target to the entrance pupil of the instrument. By inserting an imaging lens between the target and the instrument we have effectively moved the position of the entrance pupil.

Using the Gaussian lens law again it is straightforward to show that the (real) image of the entrance pupil is located at a distance, $s_{p}$, from the imaging lens, given by

$$
s_{p}=\frac{f_{a} \omega}{\omega-f_{a}} .
$$

Consequently, the distance, $R_{p}$, from the target to the entrance pupil is given by

$$
R_{p}=\left|s_{p}-a_{o}\right| \text {. }
$$

In order to measure the overlap function it is necessary to make two assumptions. First, it is assumed that the target is uniform with isotropic scattering efficiency. Second, it is assumed that over the short target translation distances in a controlled laboratory environment, variations in atmospheric transmission losses due to target translation are negligible. With these assumptions the terms $K \beta(R) T(R)$ in Eq. (1) can be replaced by a constant, $1 / C$, and using Eq. (3) the overlap can be written:

$$
O(R)=C P(R) R_{p}^{2},
$$

where the apparent target distance, $R$, and the apparent distance from the target to the entrance pupil, $R_{p}$, are given explicitly by Eqs. (ㅁ) and ( $\underline{6}$ ), respectively.

Although the basic imaging system of Fig. 1 provides a way to measure the overlap function of a lidar instrument in a confined space, it is not easy to use in practice. Because the distance $R$ is a nonlinear function of the target distance $a_{0}$, measurements are clustered around the focal plane of the imaging lens. Furthermore, for typical lidar systems, the range correction factor $R_{p}^{2}$ depends much more strongly on the target distance than the overlap function does, and thus small errors in the target distance can introduce substantial errors in the overlap measurement. The second of these problems, and to some extent the first, may be overcome by using a second imaging lens to form the compound imaging system shown in Fig. 2.

In essence, lens $L_{b}$ acts as a relay that presents a real image of the target to lens $L_{a}$. (This image becomes the object for $L_{a}$.) The main advantage of this system is that the distance between the lenses can be adjusted so that the image of the entrance pupil of the lidar is at infinity, therefore removing the need for range correction. Again employing the Gaussian lens law, the virtual range $R$ can be calculated using Eq. (ㅁ) by

$$
R=\omega+\frac{f_{a} a_{o}^{\prime}}{f_{a}-a_{o}}
$$

where

$$
a_{o}^{\prime}=f_{b}+s_{p}-\frac{b_{o} f_{b}}{b_{o}-f_{b}} .
$$

The geometric factor from Eq. (7) can now be formulated for this system with the added relay lens. In this case the image of the entrance pupil is at infinity, so as $b$ changes, the solid angle subtended by the entrance pupil does not change. The range dependence of the geometrical factor is thereby removed, allowing Eq. (7) to be formulated as

$$
O(R)=C P(R),
$$

where the distance to the virtual image of the target, $R$, is given explicitly by Eq. (8)).

A compound imaging system similar to that described above was employed to measure the overlap of a prototype lidar system. In the following

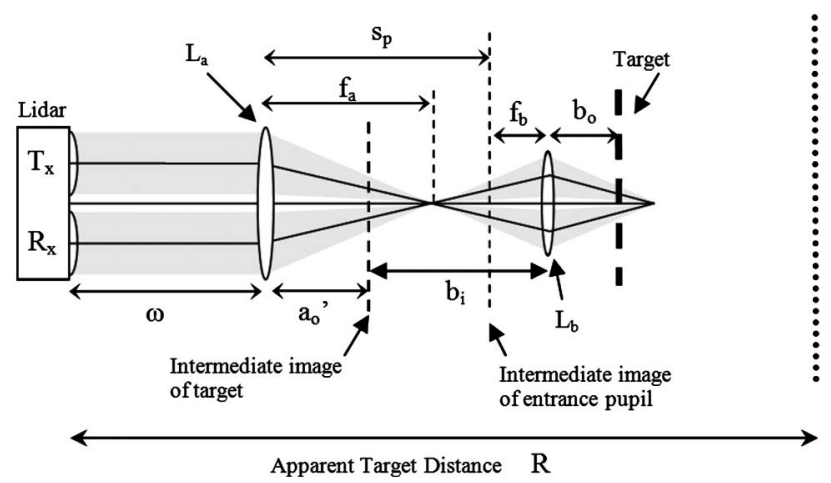

Fig. 2. Compound imaging system. 
section the lidar design is described and its geometric overlap is calculated.

\section{Lidar System and Geometric Overlap Calculation}

The prototype lidar used in this study is a biaxial system utilizing aspheric lenses of $335 \mathrm{~mm}$ focal length (at $905 \mathrm{~nm}$ ), a pulsed diode laser operating at $905 \mathrm{~nm}$, and a silicon avalanche photodiode (APD) detector as shown in Fig. 3. This instrument is designed as a ceilometer prototype to be used for measurements of cloud height and vertical visibility. It is innovative in that a single aspheric objective is divided into two elements of semicircular aperture, with optical axes separated by $21 \mathrm{~mm}$. One of the halves is used exclusively in the receiver, and the other is used exclusively in the transmitter of the biaxial lidar. The laser is tilted slightly in order to fill the transmitter half lens, but since the laser is placed at the focal point of the lens, the collimated beam leaving the instrument is vertical rather than tilted.

This design was selected for several reasons. On the transmitter side, the elliptical laser beam approximately fills a half lens without the need for beam shaping optics. On the receiver side, if the appropriate detector size for this focal length is selected, no additional aperture stop is required. The setup thus reduces the number of necessary optical components, and therefore the cost. Most importantly, the system uses two halves of the same lens, offset slightly. This combines the optical isolation benefits of a biaxial lidar with a faster overlap function similar to that of a common optics system, as the axes are closer together in this system than in a system with two comparably sized circular lenses. As noted by Stelmaszczyk et al.[16], close proximity of optical axes is desirable for biaxial instruments requiring overlap at low altitudes.

In the following, the geometric overlap of the lidar is calculated following a method similar to those mentioned previously [12-16]. However, here the calculation is derived specifically for the unique divided

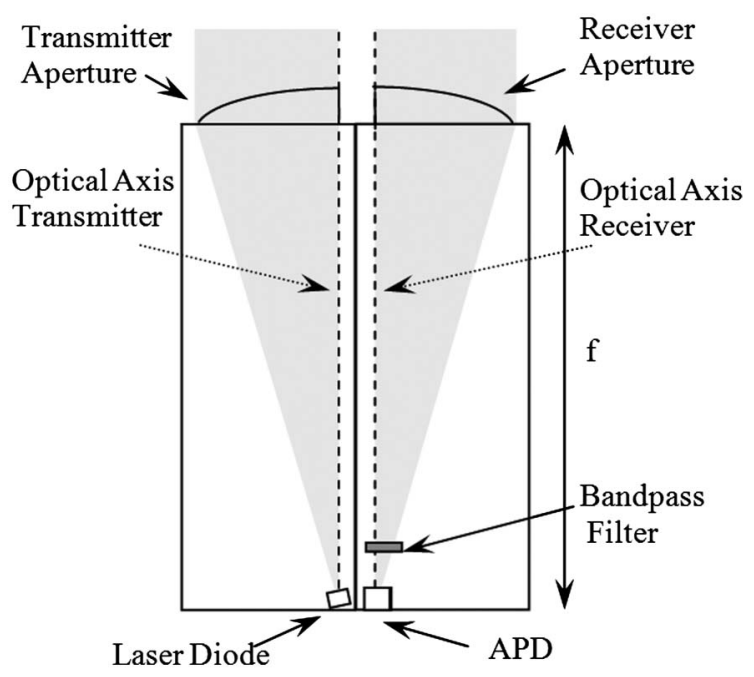

Fig. 3. Schematic of the lidar system. lens geometry and laser diode used in the prototype instrument.

The overlap, $O(s)$, of a given instrument in a plane of interest situated a distance $s$ from the exit pupil or equivalently the plane of the objective, can be written as the integral

$$
O(s)=\int_{-\infty}^{+\infty} \int_{-\infty}^{+\infty} R_{s}(x, y) T_{s}(x, y) \mathrm{d} x \mathrm{~d} y,
$$

where $R_{s}(x, y)$ and $T_{s}(x, y)$ are functions that describe, respectively, the relative sensitivity of the receiver and the intensity due to the transmitter at a point $(x, y)$ in the plane.

If the objective lenses are assumed to be ideal and diffraction effects are neglected (the validity of these assumptions will be discussed later), then the functions $R_{s}(x, y)$ and $T_{s}(x, y)$ can be estimated using a straightforward geometric approach. For the receiver, the relative sensitivity is a function of the apertures defined by the detector and the objective lens. If the detector aperture is situated in the focal plane and is small, there is a small angular divergence. Each point on the detector can then be considered as receptive to light scattered anywhere within a column, which has a cross section equal to the transmitting aperture of the receiver objective, as shown in Fig. 4 .

If the aperture of the receiver objective is defined by $A_{R}\left(x_{l}, y_{l}\right)$ and the detector aperture by $D\left(x_{f}, y_{f}\right)$ in the focal plane, then it is straightforward to show that the receiver sensitivity is given by the convolution

$$
\begin{aligned}
R_{s}(x, y)= & R_{0} \int_{-\infty}^{+\infty} \int_{-\infty}^{+\infty} D\left(\frac{f \varepsilon}{s}, \frac{f v}{s}\right) \\
& A_{R}\left(x-x_{0}-\varepsilon, y-v\right) \mathrm{d} \varepsilon \mathrm{d} v .
\end{aligned}
$$

Here $x_{0}$ is the lens offset, the factor $s / f$ is the geometric magnification, $(\varepsilon, v)$ are dummy variables of integration, and $R_{0}$ is a normalization constant such that $R_{0} \int_{-\infty}^{+\infty} \int_{-\infty}^{+\infty} R_{s}(x, y) \mathrm{d} x \mathrm{~d} y=1$. In this case, the receiver aperture is a semicircle of radius, $r_{l}=75 \mathrm{~mm}$ in the half plane $x_{l}>0$, and consequently can be described by the function

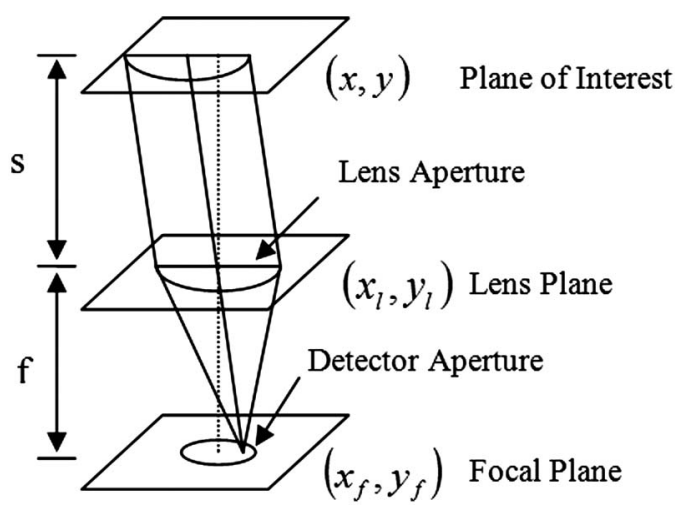

Fig. 4. Receiver geometry. 


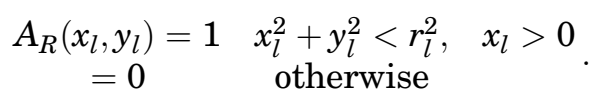

The detector has a circular aperture of radius $r_{d}=$ $0.25 \mathrm{~mm}$ and is described by the function

$$
\begin{array}{cc}
D\left(x_{f}, y_{f}\right)=1 & x_{f}^{2}+y_{f}^{2}<r_{d}^{2} \\
=0 & \text { otherwise }
\end{array}
$$

A similar approach can be used to estimate $T_{s}(x, y)$, the intensity due to the transmitter in the plane of interest by

$$
\begin{aligned}
T_{s}(x, y)= & T_{0} \int_{-\infty}^{+\infty} \int_{-\infty}^{+\infty} L\left(\frac{f \varepsilon}{s}, \frac{f v}{s}\right) \\
& A_{T}\left(x+x_{0}-\varepsilon, y-v\right) \mathrm{d} \varepsilon \mathrm{d} v,
\end{aligned}
$$

where $L\left(x_{f}, y_{f}\right)$ is the near field output of the laser source that is observed in the focal plane of the transmitter objective and $T_{0}$ is a normalization constant such that $T_{0} \int_{-\infty}^{+\infty} \int_{-\infty}^{+\infty} T_{s}(x, y) \mathrm{d} x \mathrm{~d} y=1$.

Since the light output by the laser is partially coherent, the far-field output of the laser diode is elliptical and does not fill the aperture of the lens uniformly. In this work it is assumed that the far-field laser output has an elliptical Gaussian distribution which is blocked or transmitted in the plane of the objective according to the lens aperture. Accordingly, the effective aperture of the transmitter $A_{T}\left(x_{l}, y_{l}\right)$ can be written as

$$
\begin{aligned}
A_{T}\left(x_{l}, y_{l}\right) & =e^{-\frac{1}{4}\left[\left(\frac{x_{l}-x_{g}}{\sigma_{x}}\right)^{2}+\left(\frac{y_{1}}{\sigma_{y}}\right)^{2}\right]} & x_{l}^{2}+y_{l}^{2}<r_{l}^{2}, & x_{l}<0 . \\
& =0 & & \text { otherwise }
\end{aligned}
$$

Here $\sigma_{x}=30 \mathrm{~mm}, \sigma_{y}=51 \mathrm{~mm}$, and the beam offset is $x_{g}=18 \mathrm{~mm}$ according to the measurements of the laser beam profile at the transmitter lens. Finally, the laser diode used in this case is a stacked device with three active regions. Accordingly, the near-field laser output described by $L\left(x_{f}, y_{f}\right)$ is modeled by

$$
\begin{aligned}
& L\left(x_{f}, y_{f}\right)=1 \quad\left|x_{f}\right|<\frac{L}{2}, \quad \frac{-W}{2}<y_{f}<\frac{+W}{2} \\
& =1 \quad\left|x_{f}\right|<\frac{L}{2}, \quad \frac{-W}{2}<y_{f}-S<\frac{+W}{2} \\
& =1 \quad\left|x_{f}\right|<\frac{L}{2}, \quad \frac{-W}{2}<y_{f}+S<\frac{+W}{2} \\
& =0 \text { otherwise }
\end{aligned}
$$

where the parameters $L=0.235 \mathrm{~mm}, \quad W=$ $0.001 \mathrm{~mm}$, and $S=0.005 \mathrm{~mm}$ are the length, width, and separation of the active regions, respectively, taken from the manufacturer specification. The lidar parameters were substituted into these equations and the geometric overlap function calculated according to Eq. (11) is shown in Fig. 5 as a solid line. Here $1 \%$ overlap is reached at a range of $45 \mathrm{~m}, 50 \%$ overlap is reached at $130 \mathrm{~m}, 99 \%$ overlap is reached at $285 \mathrm{~m}$, and $100 \%$ overlap is reached at $405 \mathrm{~m}$ from the instrument. The theoretical overlap of the instrument was then compared with experimental results, as described in the following section.

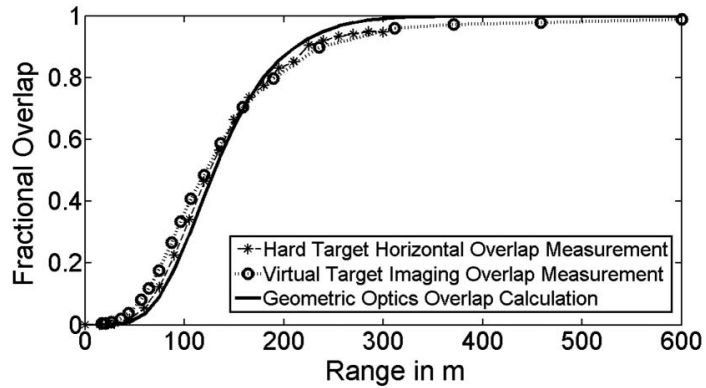

Fig. 5. Measured and calculated overlap of the prototype lidar instrument.

\section{Experimental Measurements of Overlap}

The experimental setup used to measure the lidar overlap function using the technique described in Section 2 has the same basic layout as the configuration presented in Fig. 2. It is clear, however, that in order for the system to provide an accurate measure of overlap, the aperture of any optics used must not restrict the system aperture and any aberrations introduced by additional components should have a negligible effect. In this work, the large imaging lens $L_{a}$ of the compound imaging system of Fig. $\underline{2}$ was replaced by a $0.25 \mathrm{~m}$ diameter spherical mirror with a focal length $f_{a}=3.057 \mathrm{~m}$. Consequently, the mirror was tilted at an angle of $1.5^{\circ}$ to fold the imaging system, as shown in Fig. $\underline{6}$, placing a $3^{\circ}$ angle between the light directly from the lidar and the light reflected by the mirror.

The relay lens is an achromatic doublet of nominal focal length $f_{b}=0.4 \mathrm{~m}$ and diameter of $75 \mathrm{~mm}$ (Edmund Optics NT45-419). As the calculation of virtual target distance is quite sensitive to this focal length, careful determination of the focal length of the relay lens is necessary, and this was found to be $f_{b}=$ $0.40209 \mathrm{~m}$ at a wavelength of $905 \mathrm{~nm}$. The $1.5^{\circ}$ angle was chosen to be as small as possible to reduce aberration but sufficient to allow the lidar beam to pass unobstructed to the mirror. The gap between the lidar and the mirror, $s_{o}$, was set to $20.05 \mathrm{~m}$ to ensure that the returned signal could be separated from electronic noise generated by the laser pulse. The distance between the mirror and the relay lens was adjusted such that the image of the lidar entrance pupil as viewed from the target was located at infinity, and was therefore equal to the sum of the entrance pupil image distance, $s_{p}$, and the relay lens focal length, $f_{b}$.

To ensure that aberrations were negligible, the system was modeled using OSLO optical design

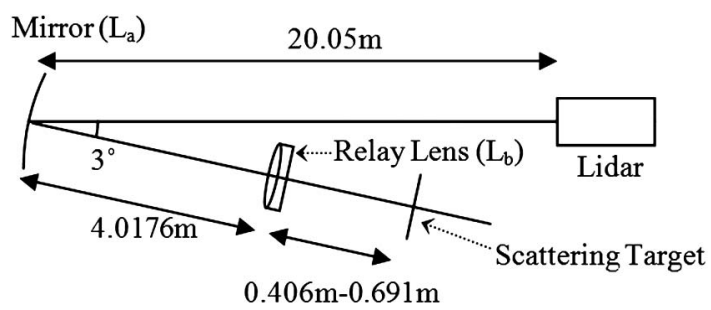

Fig. 6. Experimental setup. 
software. It was found that at the most aberrationsensitive point, where the target is placed such that its virtual image is set at infinity, the calculated minimum radius of the focused spot image of an ideal collimated laser beam was $0.031 \mathrm{~mm}$ (approximately twice that of the diffraction-limited value). At this target distance (well past the point of full overlap, so the laser image should be well within the detector image), the radius of the image of the actual laser was calculated to be $0.77 \mathrm{~mm}$ and the radius of the image of the detector was calculated to be $1.64 \mathrm{~mm}$. The influence of the $0.031 \mathrm{~mm}$ minimum spot radius should be insignificant, increasing the laser image radius by $4 \%$ and the detector image radius by $2 \%$, therefore the level of aberration present in the imaging system was judged to be acceptable.

A matte black painted aluminum target and a brown paper target were used for this work. It was assumed that these targets provided scattered returns that were largely isotropic over the range of accepted target angles. At each measurement point the difference between the returns from the brown and black targets was measured to compensate for any spurious signals arising from scattering within the imaging system. In all measurements the laser was operated at normal operating power to ensure its beam profile matched that used in the field, and lidar returns were measured from each target at each distance. Because the signals returned were significantly larger than those obtained from clouds, no reverse voltage was applied to the APD, so it behaved as a simple photodiode. This mode of operation is not expected to change the overlap characteristics of the lidar system. The target was mounted on a precision motorized translation stage, and measurements were taken at target positions ranging from 0.406 to $0.691 \mathrm{~m}$ from the back surface of the relay lens, which corresponded to lidar ranges from 17 to $8402 \mathrm{~m}$.

Figure 5 shows the overlap measured by the virtual target imaging technique as a function of range as a bold dashed line with circles. An additional measurement of the overlap was performed by gathering hard target measurements at ranges up to $300 \mathrm{~m}$ with the lidar pointing horizontally along a local air field. These results, range-corrected, are shown in Fig. 5 as a thin dashed line with asterisks. The data set gathered by the imaging technique was normalized to 1 at $8402 \mathrm{~m}$, essentially infinity, and the range-corrected horizontal hard target measurements were normalized to the imaging data at $300 \mathrm{~m}$, the farthest point that could be taken in the air field measurement. The results gathered by the imaging technique showed good agreement with both the calculated geometrical optics overlap function and the horizontal hard target overlap measurement. We would not expect the measurements to agree exactly with the calculation as the optical system was not fully characterized. The assumption, for the purposes of calculation, of aberration-free optics in the lidar prototype, is of course a tenuous one. The earlier onset of the experimental data sets could be explained by aberrations that were not accounted for or by incidental scattering in the lidar system. The fact that the experimental data sets take longer to reach full overlap than the calculated curve suggests a possible unintentional tilt of the laser away from the receiver. Berezhnyy [19] reported that diffraction effects of the laser source can have considerable influence on the overlap function of a lidar system with an annular beam shape; however, as the beam shape of this system is not annular, the Gaussian laser distribution assumption was deemed to be reasonable and diffraction effects are not expected to contribute significant error beyond the more substantial errors likely to be caused by other factors, such as uncharacterized aberrations.

If we consider the hard target overlap measurement to be our best reference for actual overlap of the system, the imaging system measurement should agree closely with it. The largest potential sources of error in the imaging method are the focal length of the relay lens (which was measured carefully in the laboratory at $633 \mathrm{~nm}$ and then recalculated at $905 \mathrm{~nm}$ based on the lens design), the focal length of the mirror (also measured in the laboratory), and the measurement of the distance of the target from the lens. Error in any of these shifts the virtual target distance to stretch or compress the $S$-shape of the typical overlap function. Uncertainty in each of these parameters was estimated to be approximately $0.1 \%$. At a range of $150 \mathrm{~m}$ (approximately half the distance to full overlap for this system at a calculated overlap of $64 \%$ ), a $0.1 \%$ error in the focal length measurement of the lens would shift the virtual target distance $4 \mathrm{~m}$, while a $0.1 \%$ error in either the focal length measurement of the mirror or the measurement of target distance would shift the virtual target distance by $3 \mathrm{~m}$ at the same point. Less shift at closer ranges and more shift at farther ranges would be expected due to the fact that the virtual target distance, $R$, is a nonlinear function of the relay lens to target distance $b_{0}$. Errors in these three parameters could account for at least some of the discrepancy between the hard target and imaging method overlap measurements. Error could also result from one of two assumptions made in this work, that the target scattering is isotropic over the accepted angle of the optical system, and that the spatial response of the APD detector is the same regardless of bias voltage.

\section{Conclusions}

A compound imaging system for the measurement of lidar overlap in the laboratory has been designed and proved. In essence, the method presents to the lidar instrument a virtual image of a scattering target at a specified range, and the response is measured. By using a compound lens system, it is possible to ensure that the entrance pupil of the instrument as seen from the scattering target is presented at infinity. In this case, the signal returned to the lidar is not diminished by the inverse square characteristic and becomes a direct measure of the overlap function. 
The measurement method has been used to characterize the overlap of a prototype lidar system. The geometric overlap of this system has also been defined and calculated. The measured results show good agreement with geometric overlap calculations. These results also show good agreement with horizontal hard target measurements of the overlap profile of the same lidar system that were made in order to provide a second reference point.

It is clear from our studies that the overlap of the geometric form factor is influenced by factors that are largely unknown and difficult to measure in practice. For example, the far-field distribution of the laser has greater influence than the aperture of the objective lens on the overlap function and in addition, this parameter can vary significantly with the output power. Similarly, the angular response of the detector, which was assumed to be uniform in this study, is likely to vary in practice, especially when combined with a bandwidth restricting interference filter. This again will modify the effective aperture of the receiver and in turn affect the overlap function.

If reliable estimates of aerosol distributions or visibility are required at close ranges then it is necessary to calibrate the lidar in this region. The measurement system discussed in this paper shows that this is indeed possible. A portable calibration instrument based on the generic compound imaging system of Fig. 2 could also be envisaged. From our studies we have found that differential measurements using alternating dark and light targets provide an effective means to distinguish valid signal from noise and would allow a measurement system with a significantly shortened path to function. The main obstacle to the development of a compact, mobile instrument would be the cost of imaging optics with large aperture and suitably low f-number. This requirement is exactly the same as that of the imaging system in a compact, large aperture lidar; however, although expensive, it should not be prohibitive.

The authors would like to thank Andrew Wormald and Rahul Mandal of Loughborough University, and Michael Dunn at Campbell Scientific, Ltd. for their assistance with conducting this experiment.

\section{References}

1. M. Ligda, "Meteorological observations with a pulsed laser radar," in Proceedings 1st Conference on Laser Technology (U.S. Office of Naval Research, 1963), pp. 63-72.

2. G. Fiocco and G. Grams, "Observations of the aerosol layer at $20 \mathrm{~km}$ by optical radar," J. Atmos. Sci. 21, 323-324 (1964).

3. U. Wandinger, "Introduction to lidar," in LIDAR: RangeResolved Optical Remote Sensing of the Atmosphere, C. Weitkamp, ed. (Springer, 2005), pp. 1-18.

4. J. D. Klett, "Stable analytical inversion solution for processing lidar returns," Appl. Opt. 20, 211-220 (1981).

5. V. Kovalev and W. Eichinger, Elastic Lidar: Theory, Practice, and Analysis Methods (Wiley, 2004).

6. V. Simeonov, G. Larcheveque, P. Quaglia, H. van den Bergh, and B. Calpini, "Influence of the photomultiplier tube spatial uniformity on lidar signals," Appl. Opt. 38, 5186-5190 (1999).
7. E. M. Patterson, D. W. Roberts, and G. G. Gimmestad, "Initial measurements using a $1.54 \mu \mathrm{m}$ eyesafe Raman shifted lidar," Appl. Opt. 28, 4978-4981 (1989).

8. R. E. W. Pettifer, G. J. Jenkins, P. G. Healey, and J. H. Convery, "A large coaxial lidar for elastic and inelastic scattering studies of the stratosphere," Opt. Quantum Electron. 8, 409-423 (1976).

9. C. Münkel, N. Eresmaa, J. Räsänen, and A. Karppinen, "Retrieval of mixing height and dust concentration with lidar ceilometer," Boundary-Layer Meteorol. 124, 117-128 (2007).

10. J. R. Campbell, D. L. Hlavka, E. J. Welton, C. J. Flynn, D. D. Turner, J. D. Spinhirne, V. S. Scott, and I. H. Hwang, "Fulltime, eye-safe cloud and aerosol lidar observation at atmospheric radiation measurement program sites: instruments and data processing," J. Atmos. Ocean. Technol. 19, 431-442 (2002).

11. Y. Sasano, H. Shimizu, N. Takeuchi, and M. Okuda, "Geometrical form factor in the laser radar equation: an experimental determination," Appl. Opt. 18, 3908-3910 (1979).

12. T. Halldórsson and J. Langerholc, "Geometrical form factors for the lidar function," Appl. Opt. 17, 240-244 (1978).

13. K. Sassen and G. C. Dodd, "Lidar crossover function and misalignment effects," Appl. Opt. 21, 3162-3165 (1982).

14. G. M. Ancellet, M. J. Kavaya, R. T. Menzies, and A. M. Brothers, "Lidar telescope overlap function and effects of misalignment for unstable resonator transmitter and coherent receiver," Appl. Opt. 25, 2886-2890 (1986).

15. H. Kuze, H. Kinjo, Y. Sakurada, and N. Takeuchi, "Fieldof-view dependence of lidar signals by use of Newtonian and Cassegrainian telescopes," Appl. Opt. 37, 3128-3132 (1998).

16. K. Stelmaszczyk, M. Dell'Aglio, S. Chudzynski, T. Stacewicz, and L. Wöste, "Analytical function for lidar geometrical compression form-factor calculations," Appl. Opt. 44, 1323-1331 (2005).

17. R. Velotta, B. Bartoli, R. Capobianco, L. Fiorani, and N. Spinelli, "Analysis of the receiver response in lidar measurements," Appl. Opt. 37, 6999-7007 (1998).

18. P. Kokkalis, G. Georgoussis, A. Papayannis, D. Hatzidimitriou, J. Porteneuve, R. E. Mamouri, and G. Tsaknakis, "Optimization-through optical design-of a multiwavelength fiber-based Raman-lidar system in the near-field for vertical aerosol measurements in the troposphere," in Proceedings of the 8th International Symposium on Tropospheric Profiling, A. Apituley, H. W. J. Russchenberg, and W. A. A. Monna, eds. (ISTP, 2009), paper S06-P01.

19. I. Berezhnyy, "A combined diffraction and geometrical optics approach for lidar overlap function computation," Opt. Lasers Eng. 47, 855-859 (2009).

20. U. Wandinger and A. Ansmann, "Experimental determination of the lidar overlap profile with Raman lidar," Appl. Opt. 41, 511-514 (2002)

21. K. Tomine, C. Hirayama, K. Michimoto, and N. Takeuchi, "Experimental determination of the crossover function in the laser radar equation for days with a light mist," Appl. Opt. 28, 2194-2195 (1989).

22. S. W. Dho, Y. J. Park, and H. J. Kong, "Experimental determination of a geometric form factor in a lidar equation for an inhomogeneous atmosphere," Appl. Opt. 36, 6009-6010 (1997).

23. S. Hu, X. Wang, Y. Wu, C. $\mathrm{Li}$, and $\mathrm{H}$. Hu, "Geometrical form factor determination with Raman backscattering signals," Opt. Lett. 30, 1879-1881 (2005).

24. J. L. Guerrero-Rascado, M. J. Costa, D. Bortoli, A. M. Silva, H. Lyamani, and L. Alados-Arboledas, "Infrared lidar overlap function: an experimental determination," Opt. Express 18, 20350-20359 (2010).

25. W. J. Smith, Modern Optical Engineering (SPIE, 2008). 